\title{
Corrigendum to "Research on Energy-Saving Experimental of Critical Dehumidification of Combined Drying by Dehumidification Wheel and Heat Pump"
}

\author{
Jiaoling Wang $(\mathbb{D}$, Weidong Song, Chengqian Jin $(\mathbb{D}$, Tianhang Ding, Mingyou Wang, \\ and Jinji Wu
}

Nanjing Institute of Agricultural Mechanization, Ministry of Agriculture and Rural Affairs, Nanjing, China

Correspondence should be addressed to Chengqian Jin; 412114402@qq.com

Received 12 October 2021; Accepted 12 October 2021; Published 28 October 2021

Copyright ( 2021 Jiaoling Wang et al. This is an open access article distributed under the Creative Commons Attribution License, which permits unrestricted use, distribution, and reproduction in any medium, provided the original work is properly cited.

In the article titled "Research on Energy-Saving Experimental of Critical Dehumidification of Combined Drying by Dehumidification Wheel and Heat Pump" [1], Figures 5 and 6 were incorrect due to a change in the simulation conditions. The authors confirm that this change does not impact the results and conclusion of the article, and the updated figures are as follows.

In addition, there is an error in Section 3.1 of Results and Discussion where the following sentence should be corrected from:
"The reason for that decrease in the temperature should be that, although the inlet air temperature has been increased, the unit cooling capacity has been increased by a greater extent; consequently, the overall outlet air temperature is decreased."to "The reason for that decrease in the temperature should be that, although the inlet air temperature has been increased, the unit cooling capacity has been increased by a greater extent. The decrease in the relative humidity of the inlet air causes the evaporation temperature to drop." 


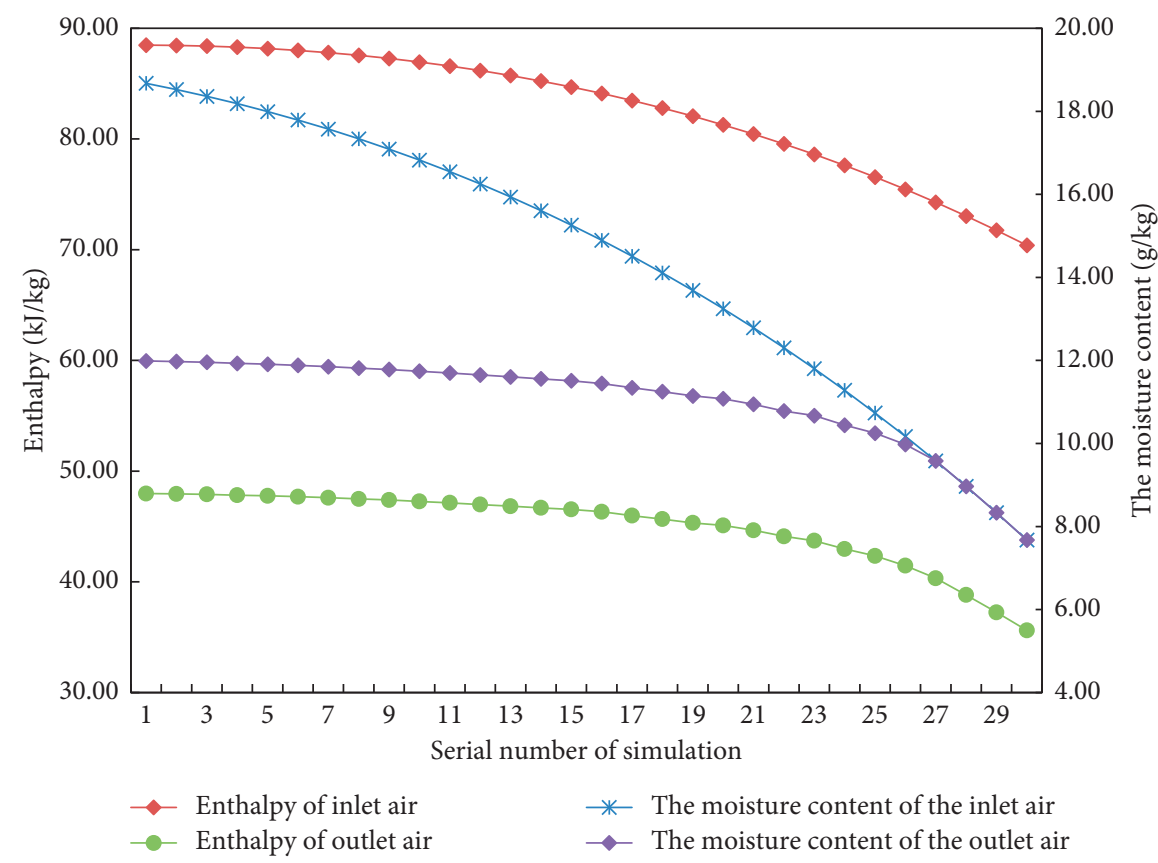

Figure 5: Enthalpy and moisture content of the inlet and outlet air of the evaporator.

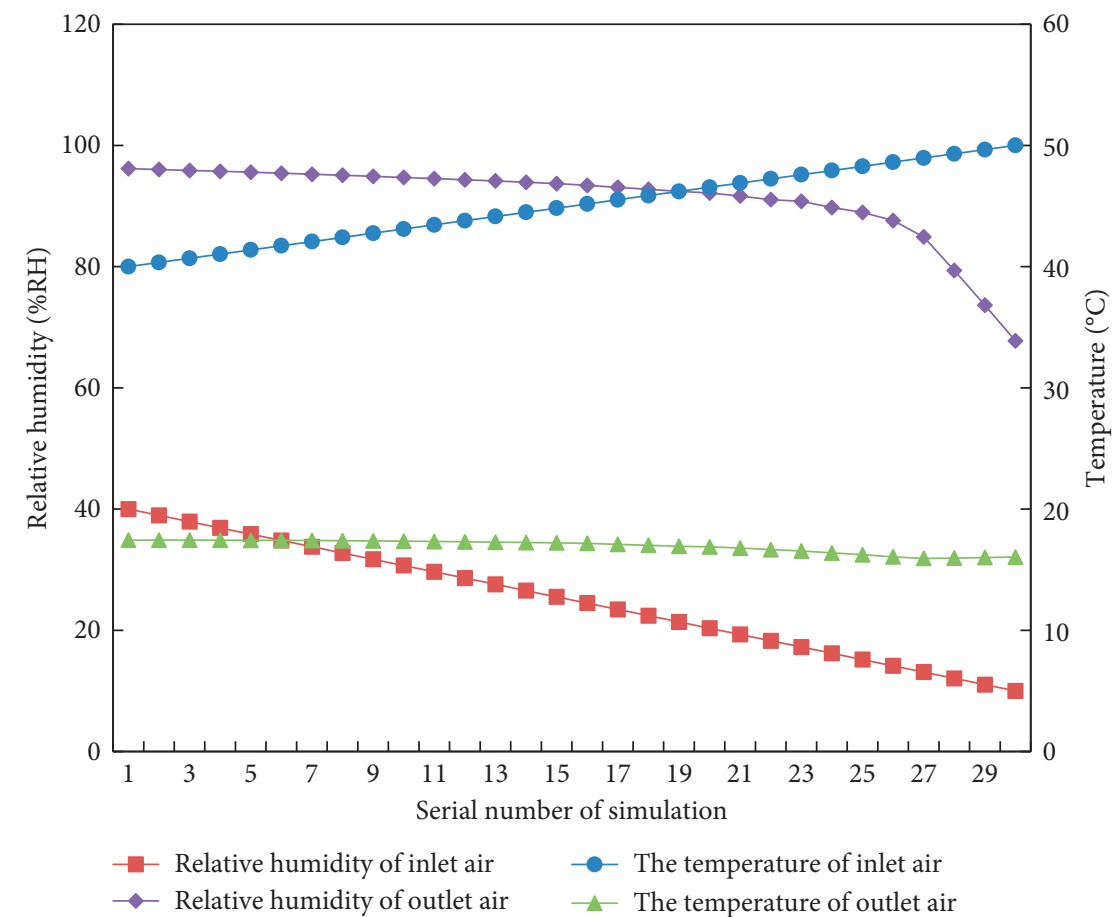

Figure 6: Temperature and relative humidity of the inlet and outlet air of the evaporator.

Finally, the Acknowledgments section should be corrected as follows.

\section{Acknowledgments}

This research was supported by the Central Public-Interest Scientific Institution Basal Research Fund (S202006-02 and
S202006-03); Independent Innovation Fund of Agricultural Science and Technology in Jiangsu Province (CX (19) 3117); Agricultural Science and Technology Innovation Program of the Chinese Academy of Agricultural Sciences-Drying and Processing Equipment Team of Special Agricultural Products (2021); and National Key Research and Development Program of China (2020YFD1000300). 


\section{References}

[1] J. Wang, W. Song, C. Jin, T. Ding, M. Wang, and J. Wu, "Research on energy-saving experimental of critical dehumidification of combined drying by dehumidification wheel and heat pump," Journal of Food Quality, vol. 2021, Article ID 6635517, 11 pages, 2021. 